\title{
Adverse impacts of mobile phone tower radiation on human health
}

\begin{abstract}
These days mobile phones have become associated with elementary a part of our life, this is often one in every of the foremost necessary mediums for communication. Consequently, several portable towers are planted to hide additional areas, particularly in huddled cities and concrete areas. Now, the bottom stations made on these locations have transceivers that employ mistreatment frequency (RF) waves to determine communication among users within the mobile network. Because of the variety of base stations needed it will increase with bigger portable use with market competition, and with new technological capabilities. The microwave frequencies utilized in mobile communication cause thermal and nonthermal effects and leave a negative impact on the biological system. The quantity of RFEMW radiation energy absorbed by human tissue depends on the frequency, intensity, polarization, and period of exposure. The EMR is recognized because of the major reason behind cancer. This review paper presents the potential biological and medicine health effects of high-intensity portable tower radiation.
\end{abstract}

Keywords: mobile phone, electromagnetic radiation, cell phone tower
Volume 7 Issue 6 - 2020

\author{
Shriyash Mohril,' Mahipal Singh Sankhla, ${ }^{2}$ \\ Swaroop S Sonone, ${ }^{3}$ Kapil Parihar, ${ }^{2}$ Rajeev \\ Kumar $^{4}$ \\ 'Process Associate, Roboticwares Pvt. Ltd. Noida, India \\ ${ }^{2}$ Research Scholars, Department of Forensic Science, School of \\ Basic and Applied Science, Galgotias University, India \\ ${ }^{3}$ Student, Forensic Science, Government Institute of Forensic \\ Science, India \\ ${ }^{4}$ Associate Professor, Department of Forensic Science, School of \\ Basic and Applied Science, India
}

\begin{abstract}
Correspondence: Mahipal Singh Sankhla, Research Scholars, Department of Forensic Science, School of Basic and Applied Science, Galgotias University, India, Email mahipal4n6@gmail.com
\end{abstract}

Received: September 22, 2020 | Published: November 09, 2020

\section{Introduction}

Nowadays, the tremendous growth in technology and smart devices has seen users get caught using them in their daily habits. Smart devices became part and parcel of our lifestyle, a feat that has turned top communication businesses to develop more advanced transmission means can cover this extensive demand. The transmission mediums are the mobile towers, which comprise antennas and base transceiver stations that use frequency (RF) to repair communication channels for users. ${ }^{1}$ People use mobile phones for communication reception, work, or anyplace their but just a couple of are concerned about their wellness indications and potential security measures. Most of the People aren't aware of mobile and cell tower radiations which are very dangerous due to electromagnetic radiation (EMR) exposure. ${ }^{2}$ Cellular wireless communications became omnipresent. Wireless technology is predicated on extensive networks of base stations that connect the users through frequency (RF) signals. Over the last decade, there has been an outstanding deal of concern about possible health consequences caused by human exposure to RF generally and radiations from base stations especially ${ }^{3}$ measures personal exposure to radiofrequency electromagnetic fields during a swiss population sample demonstrated that the quality exposure contribution from the mobile tower has relevance for cumulative long-term wholebody exposure to radiofrequency electromagnetic fields. However, needless to say, it's of minor importance for cumulative exposure to the very best of normal mobile users. ${ }^{4}$ Telephone technology has several advantages and has grown rapidly. Presently 80 crores telephone users and around 4.5 lakhs telephone towers installed to supply the communication facilities in India. The number has risen remarkably because of the discounted price of the cell phones and its portability, without recognizing its faults. "For the succeeding five years, cellphone towers are demanded to be constructed at 3 percent CAGR and thus the total number of towers is forecasted to grow to quite 511,000 by the financial year 2020, of which 30,000 towers are supposed to be only providing data sites," Deloitte India statement said. The report Indian Tower Industry: the end of the day is Data estimates that there are about 400,000 mobile towers which include only 700 standalone $3 \mathrm{G}$ and $4 \mathrm{G}$ towers. Rowley and Joyner (2012) gathered together the tower electromagnetic field (EMF) radiation data from measurement operations in 21 countries throughout the globe over a couple of years. The database of over 173,000 individual data points shows that the worldwide average exposure level from towers is as low as 0.73 mill watt per square meter, which is over 5,000 times below the WHO endorsed radiation norm for mobile towers for the frequency range considered 4 watts per square meter at $800 \mathrm{MHz} .{ }^{5}$ The bulk of those towers are positioned near the domestic and office structures to supply trustworthy mobile coverage to the users. A mobile tower and its transmitting power are designed in such a way that it covers a distance of a minimum of a couple of kilometers, implying that mobile at that distance should be ready to transmit and receive enough signal for a correct communication. A building situated at 10 's of meters from the tower will receive 10,000 times stronger signals than required for mobile communication. In cities like Mumbai, Delhi, Bangalore, etc, many folks reside within these high radiation zones. Not all standards and guidelines throughout the world have recommended equivalent limits for exposure. As an example, some published exposure limits in Russia and a few eastern European countries are generally more restrictive than existing or proposed recommendations for exposure developed in North America and other parts of Europe. ${ }^{6}$ The utilization of cell phones is increasing immensely day-by-day but most of the people (who use the cellular phone) don't skill cell phones impact human health. it's almost clear from the research that the radiation from the phone is liable for many diseases quite a brain tumor, headaches, STM loss, differing types of heart diseases, etc. within the presence of varied RF sources, including telephone handsets and broadcast antennas which contribute to the general environmental exposure have become a prominent concern about the security of this new technology and also as human health. ${ }^{7}$

\section{Cell tower radiation}

GSM900 base station antenna transmits within the frequency range of $935-960 \mathrm{MHz}$. This waveband of twenty-five $\mathrm{MHz}$ is split into twenty sub-bands of $1.2 \mathrm{MHz}$, allotted to varied operators. 
There could also be different transmitter frequencies (1 to 5) allotted to at least one operator with a better limit of $6.2 \mathrm{MHz}$ bandwidth. Transmission of 10 to $20 \mathrm{~W}$ of power is done by each carrier. So, one operator may transmit 50 to $100 \mathrm{~W}$ of power and there could also be 3-4 operators on the corresponding rooftop or tower, thereby total transmitted power maybe 200 to $400 \mathrm{~W}$. Besides, directional antennas are used, which usually may have an addition of around $17 \mathrm{~dB}$ (numeric value is 50). So effectively, several $\mathrm{KW}$ of power could also be transmitted within the main beam direction. ${ }^{8}$ The density of cell towers is directly connected to the density of the population. Also, the pattern of directional antennas is extremely crucial within the entire transmission method see (Figure 1). ${ }^{2}$

\section{Frequency ranges of towers}

Antennas on Cell tower transmit in the frequency range of:

$$
869 \text { - } 890 \mathrm{MHz} \text { (CDMA) }
$$

935 - $960 \mathrm{MHz}$ (GSM900)

$$
1805-1880 \mathrm{MHz}(\mathrm{GSM} 1800)
$$

$2110-2170 \mathrm{MHz}(3 \mathrm{G}){ }^{8}$

\section{Electromagnetic spectrum and Rf-Emf radiation}

The range of RF-EMF radiation is Ten MHz-Three Hundred GHz. Mobile phone technologies utilize frequencies principally between Eight Hundred MHz and Three GHz, Four GHz and cell tower aerials use a frequency of Nine Hundred or Eighteen Hundred MHz, pulsed at low frequencies, usually known as microwaves (Three Hundred $\mathrm{MHz}-$ Three Hundred GHz) frequency. ${ }^{5}$

\section{Electromagnetic radiation and types}

Electromagnetic radiation could also be a kind of energy showing wave-like behaviour because it progresses within space. The electromagnetic stream has both magnetic and electric elements, which sway in state perpendicular to them and to the way of energy propagation. An electromagnetic wave is often categorized into ionizing radiation and non-ionizing support whether it's able to ionize atoms \& demolishing chemical bonds. Non-ionizing emission is correlated with 2 major possible risks: biological and electrical. Remarkably large power radiation can produce electrical flows powerful enough to form sparkles (electrical arcs) when an induced charge surpasses the division voltage of the encircling medium. These flashes can then inflame combustible substances or gases, likely resulting in an outburst ${ }^{9}$ Magnetic areas affect flowing currents within the physical body and therefore the strength of those magnetic flux depends directly on the power of the magnetic field. These currents produce venations and tissues to stimulate which successively influences physiological processes. The impact of the faint EM radiations on an individual is often understood as a series of events which involves susceptibility to EM radiations which if received accentuates the physiological field patterns, an increase of energy and information into the liquid body substance, change within the functional movements of the cell which finally results into some disease. ${ }^{10}$ The phone possesses to emit frequency energy at levels adequate to obtain base stations (antenna towers). As the energy is transmitted as microwaves inside the proximity of the user's head there exists cares about the security of this technology. There are articles from cell studies also from animal studies that there could also be a notable risk of forming cancer tumefactions, leading brain tumours within the user of phones. Counting on the extent of disclosure, radiation can unfavourably influence people directly and their generations indirectly. Transmission can harm cells of the body, enhancing the danger of cancer or serious hereditary variations which will be passed on to forthcoming generations; or, if the volumes are abundant to cause extensive tissue injury, which is going to cause mortality within a couple of weeks of vulnerability. ${ }^{11-13}$ Electromagnetic radiation can be categorized on the ability of a single photon possessing energy greater than $10 \mathrm{eV}$ to ionize oxygen or eradicate chemical bonds. Types are Ionizing radiation and Nonionizing radiation. ${ }^{14}$

1. Ionizing radiation: EMR with sufficient energy to generate ions by combining with matter (about $10 \mathrm{eV}$ in biological systems). Examples are ultraviolet rays, X-rays, gamma rays, cosmic rays, etc. Primary health effects from ionizing radiation are damage to living tissues (acute) and alternations in the molecular structure which changes cell genetic tissue (chronic). ${ }^{5}$

2. Non-ionizing radiation: RF energy is identified as non-ionizing radiation. The photon energies of $\mathrm{RF}$ electromagnetic waves are not adequate to produce the ionization of atoms and molecules. Examples are radio waves, microwaves, infrared waves, etc. The primary health effect of non-ionizing radiation is temperature production in body tissue. ${ }^{5}$

\section{Radio frequency radiation}

RF energy in the wavelength spectrum of LF, MF, HF VHF, UHF, or Microwaves is usually attributed to radio waves, RF radiation, or $\mathrm{RF}$ radiations. For this discussion, the term "RF energy" is used for all frequencies between $30 \mathrm{kHz}$ and $300 \mathrm{GHz}$. Some associated facts concerning RF energy are:

I. The physiological effects of RF energy are equivalent to the rate of energy consumption, and the level of consumption varies light with frequency.

II. RF energy can heat human tissue, like the way that microwaves heat food, and can be dangerous if the expression is adequately severe or extended. Injury to tissue may be produced by susceptibility to high levels of RF energy because the body is not outfitted to consume the unnecessary volumes of heat generated. Potential injuries include skin burns, deep burns, heat depletion, and heatstroke. Eyes are especially exposed to extensive disclosure to RF energy; the lack of blood flow to cool the cornea can produce cataracts..$^{15}$

\section{Effects on human health}

Biological effects: If an individual is exposed to electromagnetic radiation, it receives radiation, because the body is $70 \%$ fluid. It is likely to cook in the microwave furnace. There will be varied vibrations of broadcasting frequencies within the body, which generates localized warming within the body. This leads to boils, dehydration of fluids nearby eyes, joints, brain, abdomen, heart, etc. ${ }^{6}$ People diverge in their reply to related levels of EMF radiation. For few people, smalltime outcomes from cell tower transmission disclosure may include headaches, sleep disturbances, reduced memory, psychic excitation, nervousness, stress, distress, hunger distress, and lethargy. ${ }^{16}$ The current international rules (based on ICNIRP references) are solely based upon the thermal consequences of radiation whereas several epidemiological and empirical studies have proved to have vital physiological effects far below these criteria. Non-thermal effects of Radiofrequency pollution expand across time and the hazards are more noticeable after 8 to 10 years of expression. ${ }^{17}$ The consequences are not recognized in the primary years of vulnerability as the body has 
specific security mechanisms and the burden is on the stress proteins of the body, particularly the heat shock proteins..$^{18}$ This indicates that the body identifies these electromagnetic radiations as a potential harm. A supplementary concern is that if the pressure goes on lengthy, there's a decreased rejoinder, and consequently, the cells are less defended against the loss. This is the reason, extended or chronic expressions may be dangerous, even at very low concentrations. Radiation from phone antennas has been connected with a more prominent rise in brain tumours. ${ }^{17}$

\section{Neurological effects}

Experimental studies with aides have examined whether low-level expression to RF fields correlated with phones can influence brain function and behavior. Summarized responses to pretend RF exposure cover a broad diversity of nonspecific signs. The most usually listed signs are insomnia, weakness, dizziness, digestive disorders, and attention problems. Well-controlled and -conducted double-blind research have revealed that symptoms are not associated with RF susceptibility. There are more suggestions that these marks may be produced by preexisting circumstances such as anxiety reactions occurring from bothering about perceived RF health outcomes rather than the RF exposure per se. Till today, only complex and temporary effects have been listed, and any health entanglements remain unclear and questionable. ${ }^{19}$ Expressions used in certain studies are alike to the head from mobile phone use, rather than to the much lower RF levels connected with a common public appearance from base stations. ${ }^{20}$ Studies of the proof on electromagnetic hypersensitivity have been convoyed. Comprehensive precise research recognized suitable blind or double-blind incentive studies of people probably high-strung to the residence of EMF. A meta-analysis observed no sign of an enhanced capacity to identify EMF in "hypersensitive" members. That is, it was decided that low electromagnetic fields are not expected to be causative agents for neurological manifestations. ${ }^{21-23}$ Research into potential variations in blood cells among patients reporting EMF hypersensitivity including normal patients did not discover any variations in lymphocyte rejoinder to RF from GSM transportable telephones. ${ }^{24}$ Other researchers have likewise assumed that "based on the insufficient studies available, there is no credible evidence for a connection between damaged well-being and susceptibility to mobile phone radiation". 25

\section{Carcinogens effect}

The International Agency for Research on Cancer (IARC) has labelled RF areas as "possibly carcinogenic to humans," based on inadequate proof of a potential accession in danger for brain tumours among telephone users, and lacking evidence for different kinds of cancer. IARC also remarked that vulnerability to the brain from RF fields from telephone base stations (installed on rooftops or towers) is smaller than $1 / 100$ th the expression to the brain from mobile devices. ${ }^{26}$

\section{The Environmental protection agency (EPA) states}

At extreme levels, RF energy is critical. It can ignite the body's membranes immediately. Nevertheless, these high levels are located just near specific devices, like powerful long-distance antennae. Mobile phones and wireless channels generate RF, but not through levels that create notable warming. Besides, RF energy reduces instantly over length. At sea level, disclosure to RF from origins like cell phone towers is typically very weak. Some people are concerned about implied health results, particularly on the developing brains and bodies of children. Some researchers recommend that continuous long-term use of cell phones could produce wellness conclusions. Other researches don't find any health outcomes from cell phone usage. Long-term investigations on animals exhibited to the RF found in wireless networks (Wi-Fi) have, so far, found no health effects. Scientists proceed to investigate the effects of long-term exposure to low levels of RF. Mobile phone base stations could also be freestanding pillars or mounted on existing constructions, like trees, water tanks, or tall buildings. The aerials got to be tall sufficient to satisfactorily cover the world. Base stations are normally from 50-200 feet high. Cell phones interact with nearby cell towers principally via radiofrequency (RF) streams, a sort of energy within the spectrum among FM radio waves and microwaves. Like FM radio waves, microwaves, light, and heat, they're sorts of non-ionizing radiation. This means they are indirectly damaging the DNA inside cells, which is how more powerful (ionizing) sorts of radiation like x-rays, gamma rays, and ultraviolet (UV) light are thought to be able to cause cancer. At very great levels, RF waves can heat body membranes. But the levels of energy used by cell phones and towers are much lower. When a person makes a telephone call, a sign is transmitted from the phone's aerial to the nearest base station wire. The base station replies to the present signal by designating it as an available radiofrequency channel. RF waves transport the voice message to the bottom station. The voice signals are later transferred to a switching station, which assigns the decision to its target. Voice signals are then communicated back and forth during the call. ${ }^{26}$

\section{Conclusion}

The utilization of mobile phones is rising remarkably daily but most of the individuals do not know how cell phone towers affect personal wellness. The exclusive method to overcome these contradictory features of the most advanced technologies is a replacement but by more reliable technology. Electromagnetic radiation \& radio waves are omnipresent. More exceeding communication aids are suspected, so is the artificial electromagnetic wave. It looks like there is no means to rearrange this drift. Experts and technicians must produce more reliable and more trustworthy wireless systems and gadgets. More miniature cell size, more suitable base station aerials, and other more unconventional technologies will enable coming mobile phones to radiate much lower power and make technology a true blessing. Mobile phones when running on the verge of the top of the user effect especially high levels of vulnerability to microwaves within the near field. Never earlier in history have such high expressions transpired frequently in such a great dimension of the community. Consequently, there's a matter that disclosure may produce later unfavorable health outcomes and particularly to an increase in cancer occurrence. State, society, and corporations are required to bargain in a blameworthy way with the relatively mobile phone tower to bypass domestic areas and their inherent health hazards even though the systematic body of proof is inadequate. The status is compounded by the reality that there are likewise financial benefits associated. Although, any risk evaluation and preventive actions should be based on precise areas. To achieve approval of the resulting system, there is no option in communicating these precise grounds to the society in an extremely clear way.

\section{Acknowledgments}

$$
\text { None. }
$$

\section{Funding}

None.

\section{Conflicts of interest}

Author declares that there is no conflict of interest. 


\section{References}

1. Al-Sahly, A., Hassan, MM, Al-Rubaian, M, et al. Using GIS for Measuring Mobile Tower Radiation on Human. 2018 1st International Conference on Computer Applications \& Information Security (ICCAIS). 2018;1-6.

2. Kaushal M, Singh T, \& Kumar A. Effects of Mobile Tower Radiation \& Case Studies from Different Countries Pertaining the Issue. International Journal of Applied Engineering Research. 2012;7(11):1.

3. Lalrinthara Pachuau, Zaithanzauva Pachuau. Study of Non-Specific Health Symptoms Faced by Inhabitants Exposed to High and Low Power Density from Mobile Phone Tower Radiation. Proceedings of Second IRF International Conference. 2015.

4. Frei P, Mohler E, Neubauer G, et al. Temporal and spatial variability of personal exposure to radio frequency electromagnetic fields. Environ Res. 2009;109:779-785.

5. Sharma AB, Lamba OS. A review: source and effect of mobile communication radiation on human health. Advances in Wireless and Mobile Communications. 2017;10(3):423-435.

6. Kumar N, Kumar G. Biological effects of cell tower radiation on human body. India: ISMOT, Delhi. 2009. p. 678-679.

7. Mohril S, Sankhla MS, Chaturvedi B. Hazardous Effect of Mobile Phone Radiation on Human Health-A Review. Changes, IPASJ International Journal of Electronics \& Communication (IIJEC). 2016;4(10).

8. Kumar G. Cell Tower Radiation. India; 2010.

9. Bhargavi K., KE Balachandrudu, P Nageswar. Mobile phone radiation effects on human health. International Journal of Computational Engineering Research. 2013;3(4):196-203.

10. 18th Int. Crimean Conference. Microwave Telecommunication Technology. Ukraine. 2008.

11. CTIA. Cellular Telecommunications Industry Association. Washington, DC. 2003.

12. Goodman MF, Bents FD, Tijerina L, et al. An Investigation of the Safety Implications of Wireless Communication in Vehicles. Report Summary. Department of Transportation electronic publication. 1999.

13. USEPA. Ionizing Radiation Series No. 1. US Environmental Protection Agency. 1998.

14. Federal Communication Commission. Guidelines for the environmental effects of radiofrequency radiation. 1997;93-62.
15. Zamanian A, Hardiman C. Electromagnetic radiation and human health: A review of sources and effects. High Frequency Electronics. 2005;4(3):16-26.

16. EMF Pollution. 2020.

17. Lennart Hardell, Michael Carlberg, Kjell Hansson Mild. Epidemiological evidence for an association between use of wireless phones and tumor diseases. Pathophysiology. 2009;595.

18. Dariusz Leszczynski, Sakari Joenväärä, Jukka Reivinen, et al. Nonthermal activation of the hsp27/p38MAPK stress pathway by mobile phone radiation in human endothelial cells: molecular mechanism for cancer- and bloodbrain barrier-related effects. Differentiation. 2002;70(2-3):120-129.

19. Cosquer B, Kuster N, Cassel JC. Whole-body exposure to $2.45 \mathrm{GHz}$ electromagnetic fields does not alter 12-arm radialmaze with reduced access to spatial cues in rats. Behav Brain Res. 2005;161:331-334.

20. Valberg PA, Van Deventer TE, Repacholi MH. Workgroup report: base stations and wireless networks-radiofrequency (RF) exposures and health consequences. Environmental health perspectives. $2007 ; 115(3): 416-424$.

21. Rubin GJ, Das Munshi J, Wessely S. Electromagnetic hypersensitivity: a systematic review of provocation studies. Psychosom Med. 2005;67:224-232.

22. Rubin GJ, Das Munshi J, Wessely S. A systematic review of treatments for electromagnetic hypersensitivity. Psychother Psychosom. 2006A; 75:12-18.

23. Rubin GJ, Hahn G, Everitt BS, et al. Are some people sensitive to mobile phone signals? Within participants double blind randomised provocation study. BMJ. 2006b;332:886-891.

24. Markova E, Hillert L, Malmgren L, et al. Microwaves from GSM mobile telephones affect 53BP1 and gamma-H2AX foci in human lymphocytes from hypersensitive and healthy persons. Environ Health Perspect. 2005;113:1172-1177.

25. Seitz H, Stinner D, Eikmann T, et al. Electromagnetic hypersensitivity (EHS) and subjective health complaints associated with electromagnetic fields of mobile phone communication - a literature review published between 2000 and 2004. Sci Total Environ. 2005;349:45-55.

26. Cell Phone Towers. 2020. 